$15^{\text {th }}$ International Conference on

AEROSPACE SCIENCES \& AVIATION TECHNOLOGY,

$\boldsymbol{A S A T}$ - 15 - May 28 - 30, 2013, Email: asat@mtc.edu.eg,

Military Technical College, Kobry Elkobbah, Cairo, Egypt,

Tel: +(202) 24025292-24036138, Fax: +(202) 22621908

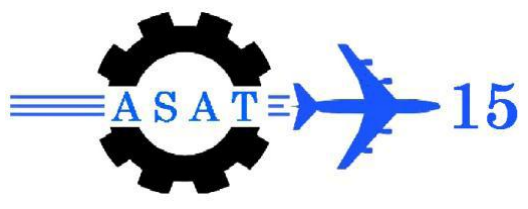

\title{
On the Modeling of Phantom AX12 Six-Legged Mobile Robot
}

\author{
A. S. Zaghloul ${ }^{*}$, W. M. Hussein ${ }^{*}$, A. Badawy ${ }^{*}$, Y. H. Hossam El-din ${ }^{\dagger}$
}

\begin{abstract}
Kinematic analysis of phantom AX12 robot is discussed in this paper. Each leg contains three revolute joints in order to mimic the structure of an insect. Denavit-Hartenberg (D-H) conventions are used to perform kinematic analysis of the six-legged robot. To develop the overall kinematic model of phantom ax12 robot, direct and inverse kinematic analyses for each leg have been considered. Trajectory of each leg is also considered for both swing and support phases. In swing phase analysis, cubic and quantic polynomials are used. The proposed model is then implemented and visualized to verify its performance in comparison with an actual phantom ax12 robot.
\end{abstract}

Keywords: Kinematic analysis, trajectory planning, six-legged.

\section{Introduction}

Walking machines are desirable because they can navigate terrain features that are similar in size to the size of the robot, whereas wheeled and tracked vehicles are only suitable for obstacles smaller than half the diameter of the wheel. Furthermore, if given an ability to find locally horizontal footholds in regionally steep terrain, they can climb extreme angles. Applications potentially include reaching territories which are unreachable or dangerous for humans, exploration, mining, military, rescue, and industrial environments, on earth and beyond. Nevertheless, legged robots are also used for experimental studies on the behavior of living animals and for testing Artificial Intelligence (AI) techniques[1].

The available options in legged robots are enormous, configuration from 2 legged (Humanoid),4 legged (Quadrupeds), 6 legged (Hexapod) and the 8 legged (Octopod) configuration like spiders[2].

Typical hexapod robots can be classified into rectangular and hexagonal ones Fig. 1. Rectangular hexapods are inspired from insects have six legs distributed symmetrically along two sides; each side having three legs, this design is fast in forward direction but less flexible in turning or moving sideways.

Hexagonal hexapods have six legs distributed axisymmetrically around the body. The hexagonal hexapod has the advantage of being more flexible in moving and achieving the same walking speeds in all directions. A lot of examples can be found of the 6 legged robot design, we will study phantom Ax12 rectangular hexapod see Fig. 2..

\footnotetext{
* Egyptian Armed Forces, Egypt.

${ }^{\dagger}$ Higher Technological Institute (HTI), Tenth of Ramadan City, Egypt
} 


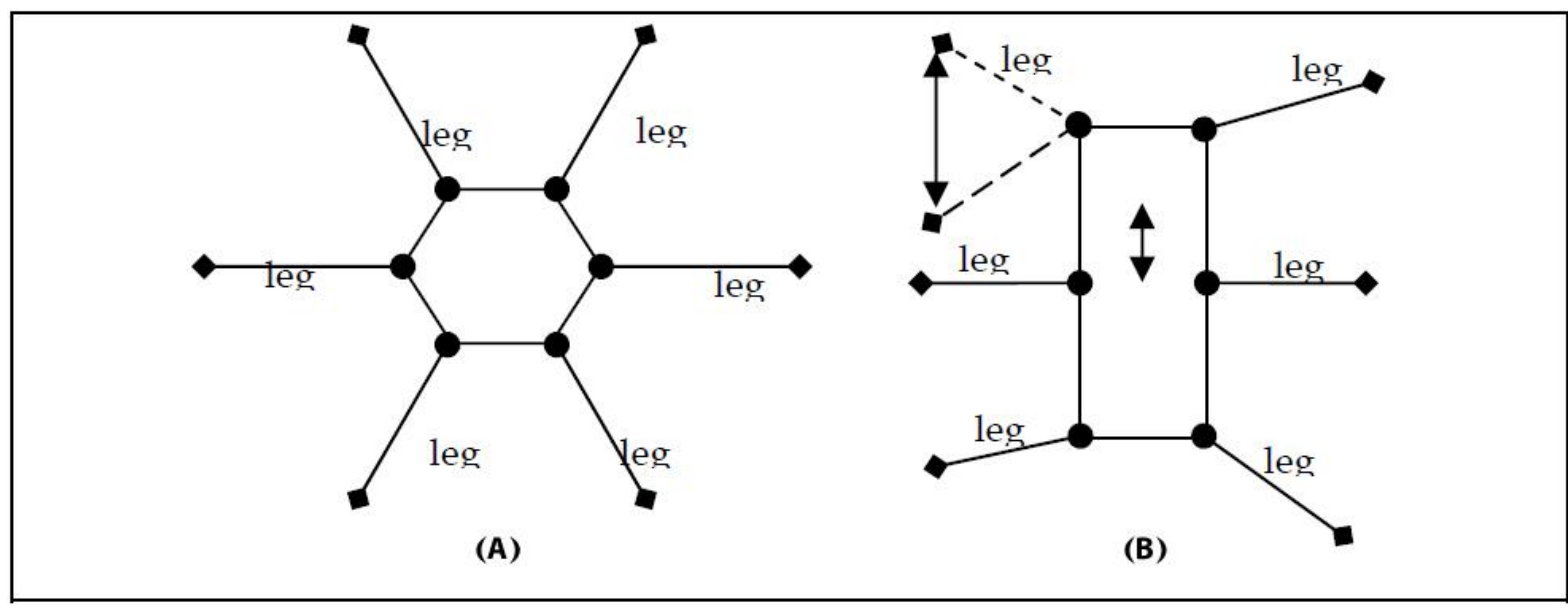

Fig. 1 Two types of hexapod robots[3]

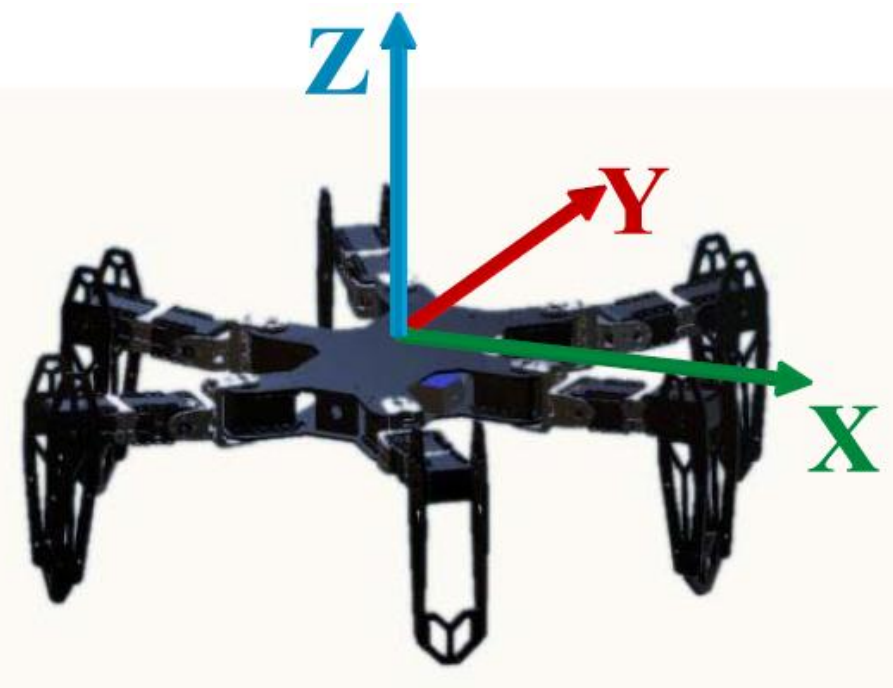

Fig. 2 3D model of phantom AX12 hexapod with the body axis at its CG

One of the main challenges in the development of hexapod robot is the locomotion system design. It involves the interaction of structures composed of prismatic or rotational joints which emulates the nature motion mechanism, allowing adapting to uneven terrain. It also needs to deal with problems like the mechanical complexity existing in legs, the mechanism stability, power consumption, synchronization of the links in each of the robots joints and the control of required number of degrees of freedom. In case of a hexapod robot with three degrees of freedom per leg it is required to synchronize eighteen degrees of freedom.

The organization of the paper is as follows: In Section 2, kinematic model of hexapod is presented. In Section 3, a leg trajectory generation algorithm for a hexapod is developed, and validation results are presented in Section 4. Section 5 concludes the paper. 


\section{Kinematic Model of Hexapod}

To be able to control the robot, kinematic model of the robot is developed. Coordinate frames are defined for all important parts of the system. The defined coordinate systems will be used throughout the paper. To be able to coordinate the movement of the robot in relation to the environment, a kinematic model is created. The kinematic model consists of both the kinematic description of the robot and its legs and an IK solution for the legs and the robot body itself. This makes it possible to calculate the joint angles for the robot legs, for a given leg and robot configuration. To derive the kinematic model, the following assumptions are made:

a) The robot moves forward in a straight path on flat surface with alternating tripod gait.

b) The trunk body is held at a constant height and parallel to the ground plane during locomotion.

c) The center of gravity of the trunk body is assumed to be at the geometric center of the body.

\section{Forward Kinematics Problem}

The forward kinematics generally means given the joint variables we get the position and orientation of the end effector in our study of phantom ax12 hexapod it means knowing the joint variables $\theta_{1}, \theta_{2}$ and $\theta_{3}$ we know the position and orientation of leg tip.

\section{Leg kinematics}

Phantom ax12 leg consists of three main links coxa, femur and tibia as shown in Fig. 3, the robotic leg, with rotational axes added. The green axis no(1) is named coxa joint at the beginning of the coxa link, the Red axis no(2) is Named femur joint at the beginning of the femur link, and the blue axis no(3) is named tibia joint at the beginning of the tibia link, each link is actuated through a Dynamixel AX-12 servo motor.

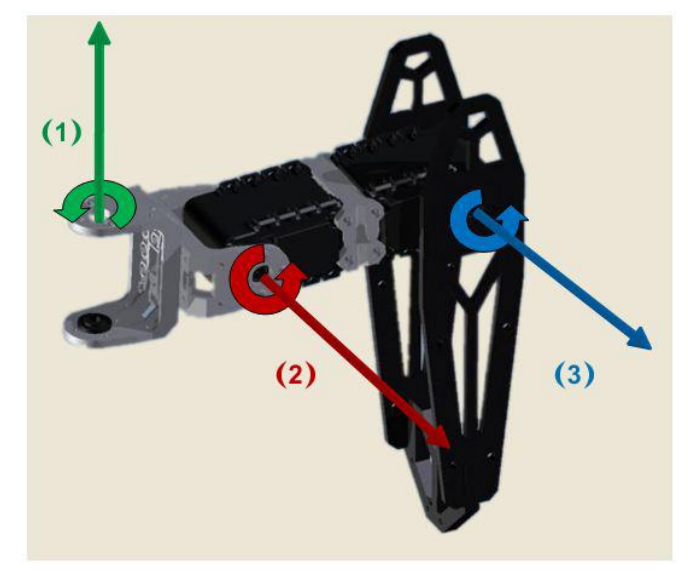

Fig. 3 The robotic leg rotational axes

Kinematic modeling of each leg using the Denavit-Hartenberg (D-H) notations[4], Fig. 4, shows the coordinate frames attached to each joint. 


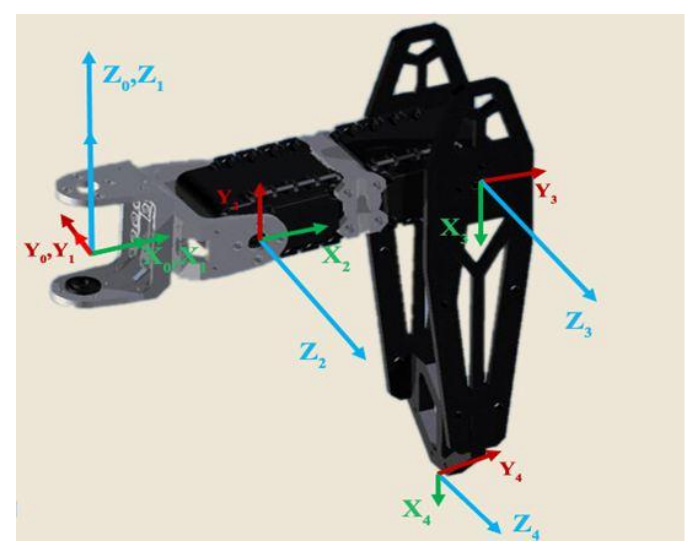

Fig. 4 One of the robot legs with all link frames

The D-H parameters of the hexapod leg are illustrated in Table 1 where:

- Link length $\mathrm{a}_{\mathrm{i}}$ is the distance from $\mathrm{Z}_{\mathrm{i}}$ to $\mathrm{Z}_{\mathrm{i}+1}$ measured along $\mathrm{X}_{\mathrm{i}}$.

- Link twist $\alpha_{i}$ is the angle from $Z_{i}$ to $Z_{i+1}$ measured about $X_{i}$.

- Link offset $d_{i}$ is the distance from $X_{i-1}$ to $X_{i}$ measured along $Z_{i}$.

- Joint angle $\theta_{\mathrm{i}}$ is the angle from $\mathrm{X}_{\mathrm{i}-1}$ to $\mathrm{X}_{\mathrm{i}}$ measured about $\mathrm{Z}_{\mathrm{i}}$.

Table 1 D-H parameters of the hexapod leg

\begin{tabular}{l|l|l|l|l}
\hline \hline Transformation/parameter & $\boldsymbol{\alpha}_{\boldsymbol{i}-\boldsymbol{1}}$ & $\boldsymbol{a}_{\boldsymbol{i}-\boldsymbol{1}}$ & $\boldsymbol{d}_{\boldsymbol{i}}$ & $\boldsymbol{\theta}_{\boldsymbol{i}}$ \\
\hline $\mathrm{\longrightarrow}$ & 0 & 0 & 0 & $\boldsymbol{\theta}_{\boldsymbol{1}}$ \\
\hline $2 \longrightarrow 1$ & 90 & 52 & 0 & $\boldsymbol{\theta}_{\boldsymbol{2}}$ \\
\hline $3 \longrightarrow 2$ & 0 & 82 & 0 & $\boldsymbol{\theta}_{\mathbf{3}}$ \\
\hline $4 \longrightarrow 3$ & 0 & 140 & 0 & 0 \\
\hline
\end{tabular}

Transformation matrix defines frame (i) relative to the frame (i - 1$)$ equals

$$
{ }^{i-1} T_{i}=\left[\begin{array}{cccc}
c \theta_{i} & -s \theta_{i} & 0 & a_{i-1} \\
s \theta_{i} c \alpha_{i-1} & c \theta_{i} c \alpha_{i-1} & -s \alpha_{i-1} & -s \alpha_{i-1} d_{i} \\
s \theta_{i} s \alpha_{i-1} & c \theta_{i} s \alpha_{i-1} & c \alpha_{i-1} & c \alpha_{i-1} d_{i} \\
0 & 0 & 0 & 1
\end{array}\right]
$$

where:

$\mathrm{c} \theta_{\mathrm{i}}=\cos \theta_{\mathrm{i}}, \mathrm{s}_{\mathrm{i}}=\sin \theta_{\mathrm{i}}$

$\operatorname{co} \alpha_{\mathrm{i}-1}=\cos \alpha_{\mathrm{i}-1}, \mathrm{~s} \alpha_{\mathrm{i}-1}=\sin \alpha_{\mathrm{i}-1}$

The specific leg transformation matrices, transforming the coordinates from one link frame to the next frame, are shown below

$$
{ }^{0} T_{1}=\left[\begin{array}{cccc}
\cos \theta_{1} & -\sin \theta_{1} & 0 & 0 \\
\sin \theta_{1} & \cos \theta_{1} & 0 & 0 \\
0 & 0 & 1 & 0 \\
0 & 0 & 0 & 1
\end{array}\right]
$$


$\begin{aligned}{ }^{1} T_{2} & =\left[\begin{array}{cccc}c \theta_{2} & -s \theta_{2} & 0 & 52 \\ 0 & 0 & -1 & 0 \\ s \theta_{2} & c \theta_{2} & 0 & 0 \\ 0 & 0 & 0 & 1\end{array}\right] \\ { }^{2} T_{3} & =\left[\begin{array}{cccc}\mathrm{c} \theta_{3} & \mathrm{~s} \theta_{3} & 0 & 82 \\ -\mathrm{s} \theta_{3} & \mathrm{c} \theta_{3} & 0 & 0 \\ 0 & 0 & 1 & 0 \\ 0 & 0 & 0 & 1\end{array}\right]\end{aligned}$

${ }^{0} T_{3}={ }^{0} T_{1} \times T_{2} \times{ }^{2} T_{3}=\left[\begin{array}{cccc}\mathrm{c}\left(\theta_{2}-\theta_{3}\right) \times c \theta_{1} & -s\left(\theta_{2}-\theta_{3}\right) \times c \theta_{1} & s \theta_{1} & c \theta_{1} \times\left(82 c \theta_{2}+52\right) \\ \mathrm{c}\left(\theta_{2}-\theta_{3}\right) \times s \theta_{1} & -s\left(\theta_{2}-\theta_{3}\right) \times s \theta_{1} & -c \theta_{1} & s \theta_{1} \times\left(82 c \theta_{2}+52\right) \\ s\left(\theta_{2}-\theta_{3}\right) & \mathrm{c}\left(\theta_{2}-\theta_{3}\right) & 0 & 82 s \theta_{2} \\ 0 & 0 & 0 & 1\end{array}\right]$

${ }^{0} T_{4}={ }^{0} T_{1} \times T_{2} \times T_{3} \times{ }^{3} T_{4}$

$=\left[\begin{array}{cccc}\mathrm{c}\left(\theta_{2}-\theta_{3}\right) \times c \theta_{1} & -s\left(\theta_{2}-\theta_{3}\right) \times c \theta_{1} & s \theta_{1} & c \theta_{1} \times\left(140 \times \mathrm{c}\left(\theta_{2}-\theta_{3}\right)+82 \times c \theta_{2}+52\right) \\ \mathrm{c}\left(\theta_{2}-\theta_{3}\right) \times s \theta_{1} & -s\left(\theta_{2}-\theta_{3}\right) \times s \theta_{1} & -c \theta_{1} & s \theta_{1} \times\left(140 \times \mathrm{c}\left(\theta_{2}-\theta_{3}\right)+82 \times c \theta_{2}+52\right) \\ s\left(\theta_{2}-\theta_{3}\right) & \mathrm{c}\left(\theta_{2}-\theta_{3}\right) & 0 & 140 \times s\left(\theta_{2}-\theta_{3}\right)+82 \times s \theta_{2} \\ 0 & 0 & 0 & 1\end{array}\right]$

\section{Body kinematics}

The position of legs on the body is defined through a transformation matrix the rotation part is only a rotation about the $\mathrm{z}$ axis with the angles of coxa point of the legs which equal (135, $180,225,315,0,45)$ relative to the order in figure of the legs (measured from the $\mathrm{x}$ axis of the body) The notations (rm), (rf) (lm) and so on Are shorthand names for the leg positions, eg. $\mathrm{rm}$ is right middle and if is left front, also the numeration of the legs are shown in Fig. 5

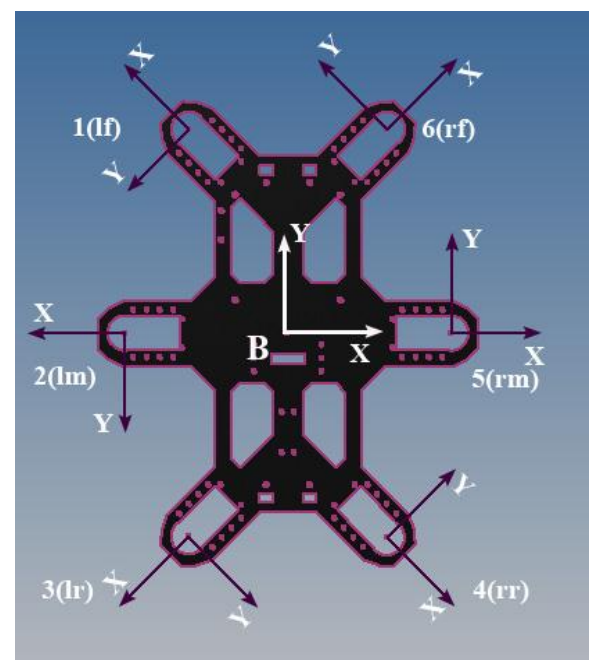

Fig. 5 The position of the leg frames relative to the robot body frame 
Transformations are denoted ${ }^{B} T_{\text {lf }}$ for the transformation from the front left leg (lf) frame to the body frame (B)

$$
{ }^{B} T_{l}=\left[\begin{array}{cccc}
\cos \gamma \kappa & -\sin \gamma \kappa & 0 & x \\
\sin \gamma \kappa & \cos \gamma \kappa & 0 & y \\
0 & 0 & 1 & 0 \\
0 & 0 & 0 & 1
\end{array}\right]
$$

where:

$\gamma_{k}$ is the angle about $\mathrm{z}$ axis of the $\mathrm{k}$ 'th leg, relative to the body frame.

The roll, pitch and yaw angles $(\alpha, \beta$ and $\gamma$ ) rotates the body around the $y$-axis, the $x$-axis and the $z$-axis correspondingly. The rotation of the body frame consists of three rotations, one about each axis. In this case the rotations occur in the yxz (roll-pitch-yaw) order

${ }^{G} T_{B}=\left[\begin{array}{cc}{ }^{G} R_{B} & { }^{G} d_{B} \\ 0 & 1\end{array}\right]=\left[\begin{array}{cccc}\cos \alpha \cos \gamma-\sin \alpha \sin \beta \sin \gamma & -\cos \beta \sin \gamma & \cos \gamma \sin \alpha+\cos \alpha \sin \beta \sin \gamma & X \\ \cos \alpha \cos \gamma+\cos \gamma \sin \alpha \sin \beta & \cos \beta \cos \gamma & \sin \alpha \sin \gamma-\cos \alpha \cos \gamma \sin \beta & Y \\ -\cos \beta \sin \alpha & \sin \beta & \cos \alpha \cos \beta & Z \\ 0 & 0 & 0 & 1\end{array}\right]$

where:

$\alpha$ is the roll angle.

$\beta$ is the pitch angle.

$\gamma$ is the yaw angle.

Now the transformation from the leg end points to the global frame can be written as

${ }^{G} T_{m 4}={ }^{G} T_{B} \times{ }^{B} T_{r m 4}$

At the end of this part if we know the joint angles and the position and orientation of the CG of the body of the hexapod we can know the position of all leg tips we build a simulator with the help of Matlab software to verify the kinematic model as shown in Fig. 6.

\section{Inverse kinematics problem}

As seen in the previous section a forward kinematic problem is solved but the reverse operation is often interesting, and if it is possible for the leg end point to reach a position in space, it is also possible to determine the angles at all the joints, for the given position To be able to find the angles of all the joints on the robot, it is necessary to know the goal position of the end points, and also the pitch, yaw, roll, and position of the CG of robot body, in the global frame. There are two main methods to solve inverse kinematics:

- Numerical solutions

Because of their iterative nature, numerical solutions generally are much slower than the corresponding closed-form solution so much so, in fact, that, for most uses, we are not interested in the numerical approach to solution of kinematics.

- Closed-form solutions

Means a solution method based on analytic expressions or on the solution of a polynomial of degree 4 or less. Within the class of closed-form solutions, we distinguish two methods of obtaining the solution:
○ Algebraic
- Geometric 


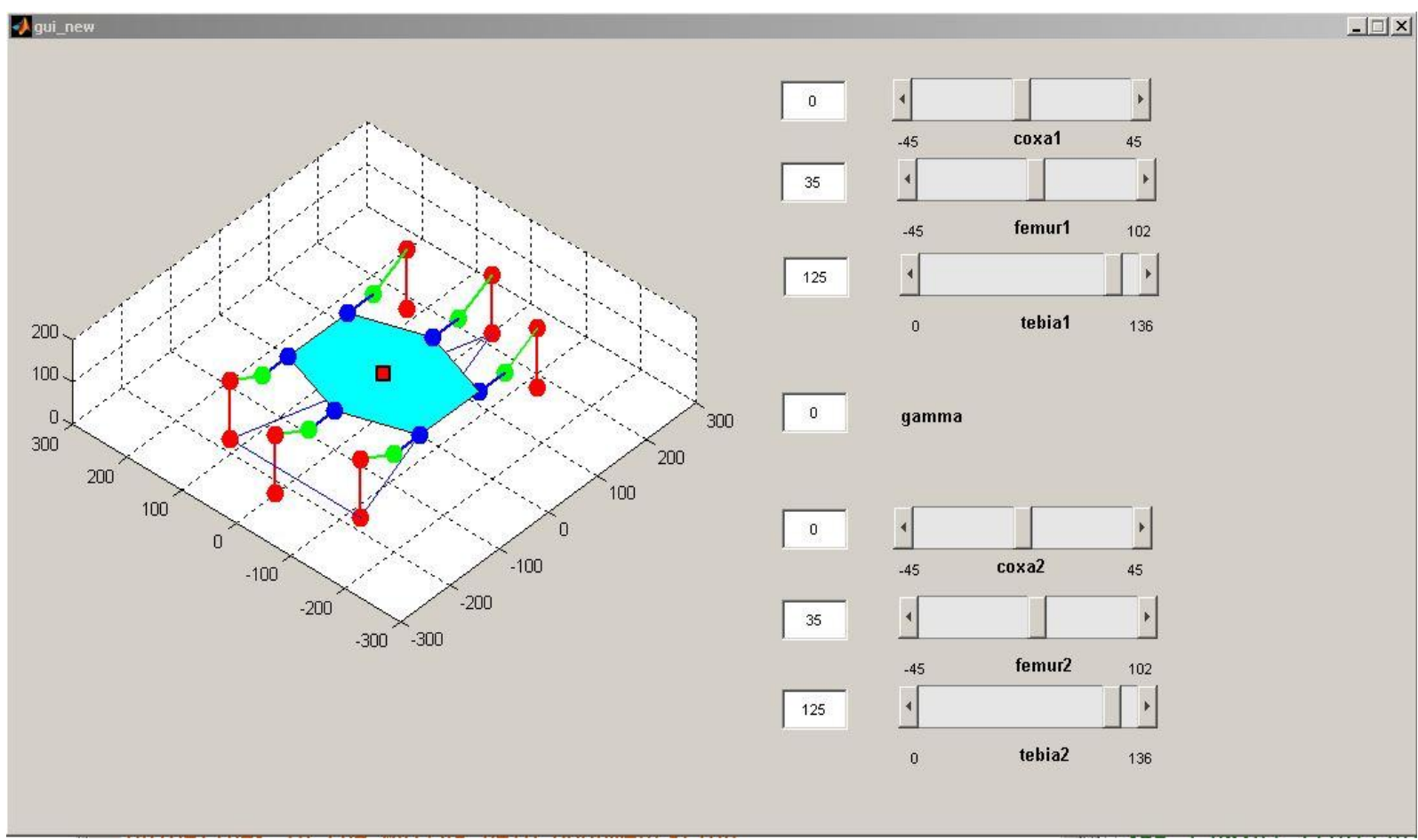

Fig. 6 Kinematic simulator

\section{Transforming from Global Frame to Leg Frame}

Before the IK can be solved for the individual legs, the leg end point coordinates, which are referenced in the global frame, needs to be transformed to the individual leg frames. This inverse transformation is the pseudo inverse of the body to leg transformation ${ }^{B} T_{l}$ and global to body frame transformation ${ }^{G} T_{B}$ pseudo inverse of ${ }^{B} T_{l}$ and ${ }^{G} T_{B}$ is shown in Equations (10) and (11).

$$
\begin{aligned}
& { }^{B} T_{G}=\left({ }^{G} T_{B}\right)^{-1}=\left[\begin{array}{cc}
\left({ }^{G} R_{B}\right)^{T} & -\left({ }^{G} R_{B}\right)^{T} \times{ }^{G} d_{B} \\
0 & 0
\end{array}\right] \\
& { }^{l} T_{B}=\left({ }^{B} T_{l}\right)^{-1}=\left[\begin{array}{cc}
\left({ }^{B} R_{l}\right)^{T} & -\left({ }^{B} R_{l}\right)^{T} \times{ }^{B} d_{l} \\
0 & 0
\end{array}\right]
\end{aligned}
$$

where:

${ }^{G} R_{B}$ is the rotational transformation from the body frame to the global frame.

${ }^{G} d_{B}$ is translational transformation from the body frame to the global frame.

${ }^{B} R_{l}$ is the rotational transformation from the leg frame to the body frame.

${ }^{B} d_{l}$ is translational transformation from the leg frame to the body frame. 


\section{Solving IK for Each Leg Geometrically}

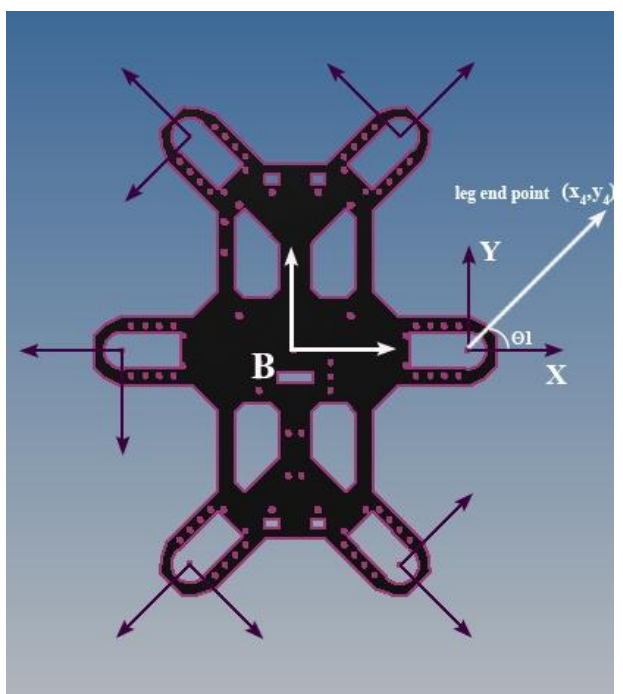

Fig. 7 The coxa joint angle, in the leg-frame

The following equations show how to solve inverse kinematics

coxa angle $\theta_{1}=\tan ^{-1} \frac{Y_{4}}{X_{4}}$

TrueX $=\sqrt{\mathrm{X}^{2}+\mathrm{Y}^{2}}-\mathrm{Lcoxa}$

$\operatorname{Im}=\sqrt{(\text { TrueX })^{2}+Z^{2}}$

Get femur angle above horizon

$\mathrm{q}_{1}=\tan ^{-1} \frac{\mathrm{Z}}{\text { TrueX }}$

Apply law of cosines in the triangle abc

$\mathrm{q}_{2}=\cos ^{-1} \frac{\sqrt{L f^{2}+\mathrm{Im}^{2}-L t^{2}}}{2 \times L f \times \operatorname{Im}}$

And tibia angle from femur

$\varphi=\cos ^{-1} \frac{\sqrt{L f^{2}+L t^{2}-\mathrm{Im}^{2}}}{2 \times L f \times L t}$

tibia angle $\theta_{3}=\varphi-180$ 


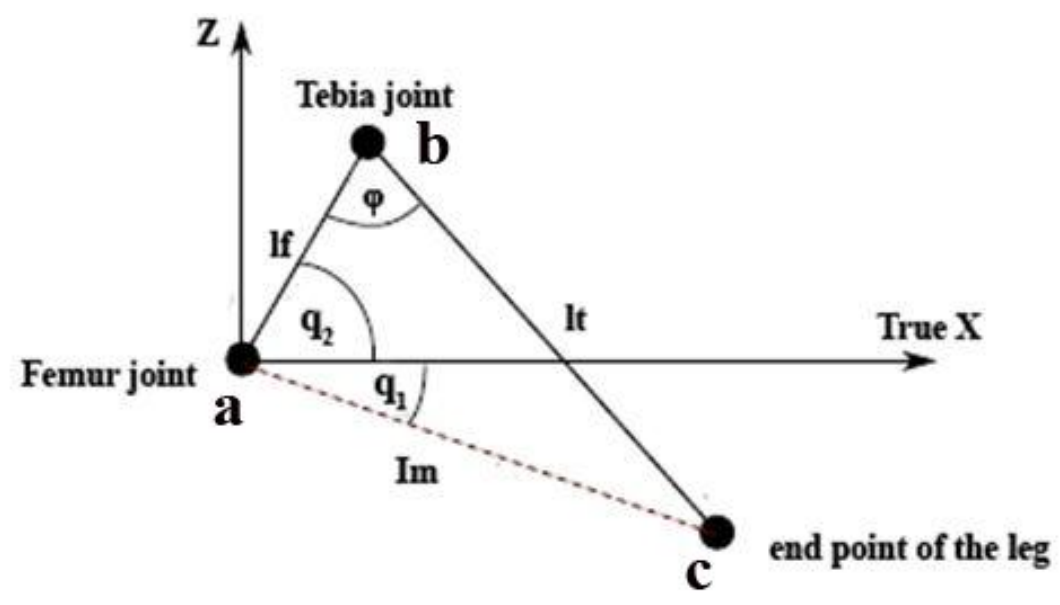

Fig. 8 The 2D triangle with vertices of the femur and the tibia link frame origins[5]

\section{Leg Trajectory Planning}

Walking speed of legged robots is also an extremely important factor for robot locomotion it depends on the number of legs (which means the more legs the robot has the speeds can achieve), the gait type and also depends on the leg trajectory of the robot

The leg trajectory will be explained in this section. All individual leg trajectories will be combined into a walking gait to move robot. The leg trajectory describes the angle of each link as a function of cycle time.

The leg trajectory parameters are: cycle time $\mathrm{T}$, duty factor $\beta$ and step size $\mathrm{L}_{\mathrm{s}}$, these parameters determine the body velocity

$\mathrm{V}_{\mathrm{n}}=\mathrm{L}_{\mathrm{s}} / \mathrm{T}$

$T_{t}=(1-\beta) T$

$T_{s}=\beta T$

where:

$\mathrm{V}_{\mathrm{n}}$ is the speed of a legged robot with $\mathrm{n}$ amount of legs performing a wave gait

Cycle time: This is defined as the time for a complete cycle of the leg locomotion of a periodic gait.

Transfer phase $T_{t}$ : The transfer phase of a leg is the period during which the foot is in the air (no ground contact).

Support phase $T_{s}$ : The support phase of a leg is the period during which the foot is on the ground and supports the robot.

Duty factor: is the ratio of the support phase and the transfer phase with respect to the Cycle time.

Gait is a description of how the robot moves its legs, there many types of gaits can the six legged robot can achieve. In our work we choose tripod gait where the robot moves two legs in one side and the middle leg on the opposite side as seen in Fig. 9. Tripod gait is the fastest gait of hexapods while keeping static stability (static stability means CG of the robot is always inside the polygon of stability). 


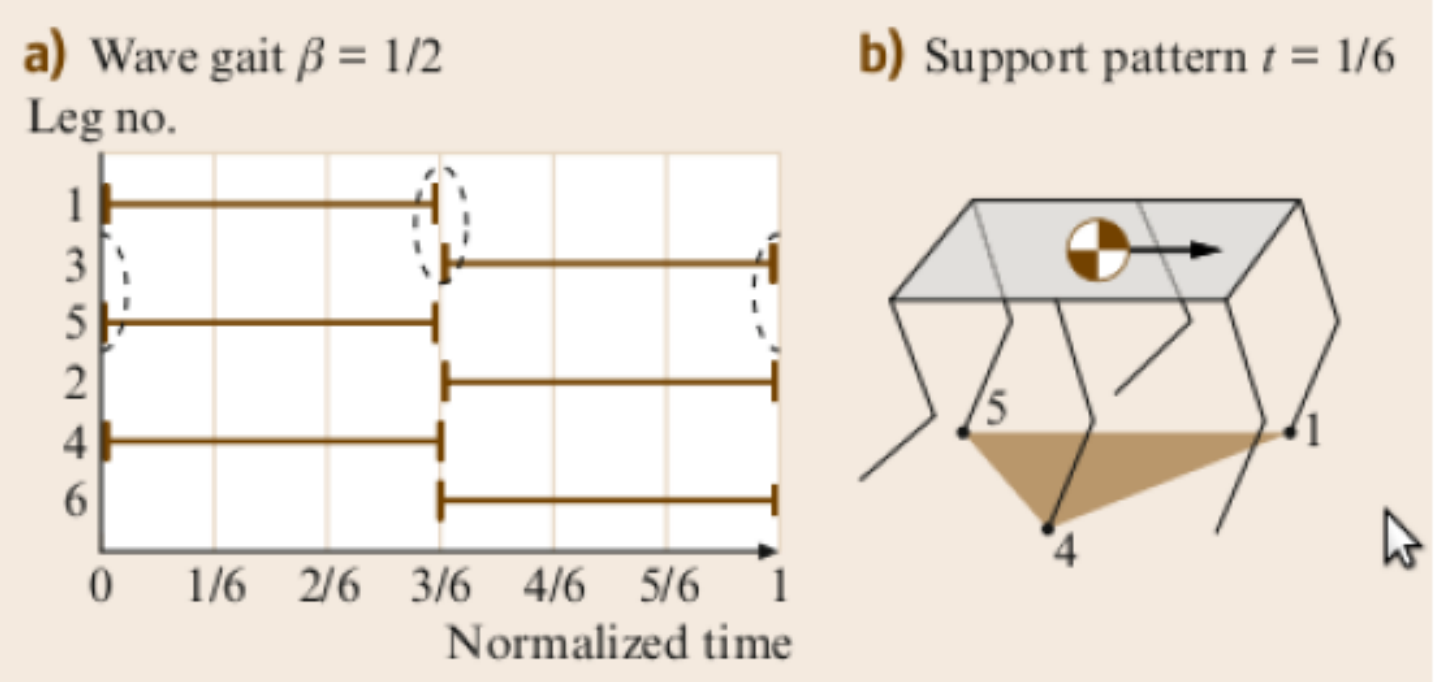

Fig. 9 Tripod gait of a hexapod robot, here three legs move at the same time while keeping static stability[2]

For our hexapod we choose the following value for simulation Gait type Tripod, Step length $26 \mathrm{~mm}$, Step height $30 \mathrm{~mm}$, (these values are taken from the real phantom Ax12 hexapod) We solve inverse kinematics at start point for the right middle leg $(219.1705,-13,0)$, via point (intermediate point) $(219.1705,0,30),(219.1705,13,0)$ (all point in the global frame, all units in $\mathrm{mm}$ ) and at end point the values of $\theta_{1}, \theta_{2}, \theta_{3}$ (in degrees) are listed in Table 2

Table 2 joint angles for start point, mid-point and end points

\begin{tabular}{c|l|c|c}
\hline \hline $\begin{array}{c}\text { Point/ } \\
\text { angle }\end{array}$ & \multicolumn{1}{c|}{$\theta_{1}$} & $\theta_{2}$ & $\theta_{3}$ \\
\hline \hline start point $\theta_{0}$ & -6.2256 & 35.0059 & 124.7146 \\
\hline via point $\theta_{\mathrm{v}}$ & 0 & 63.7989 & 141.0204 \\
\hline end point $\theta_{\mathrm{g}}$ & 6.2256 & 35.0059 & 124.7146 \\
\hline \hline
\end{tabular}

Leg trajectory can be divided into two main parts: swing and support phases.

\section{Swing (transfer) phase}

\section{Cubic polynomial}

$$
\begin{aligned}
& \theta(t)=a_{0}+a_{1} t+a_{2} t^{2}+a_{3} t^{3} \\
& \theta \cdot(t)=a_{1}+2 \times a_{2} t+3 \times a_{3} t^{2} \\
& \theta \cdot(t)=2 \times a_{2}+6 \times a_{3} t
\end{aligned}
$$

For the Calculation of the trajectory of $\theta_{1}$ (coxa angle), $\theta_{2}$ (femur angle) and $\theta_{3}$ (tibia angle) we divide the trajectory into two cubic segments where the initial angle is $\theta_{0}$, the via point is $\theta_{\mathrm{v}}$ and the goal point is $\theta_{\mathrm{g}}$ with Continuous velocity and acceleration at via point.

These constrains specify a linear equation problem having eight equations and eight unknowns; solving them we get the trajectory of each joint. For calculating Trajectory of $\theta_{1}$, $\theta_{2}$ and $\theta_{3}$ we assume $t_{f 1}=t_{f 2}=t_{f}=0.13 \mathrm{sec}$ 

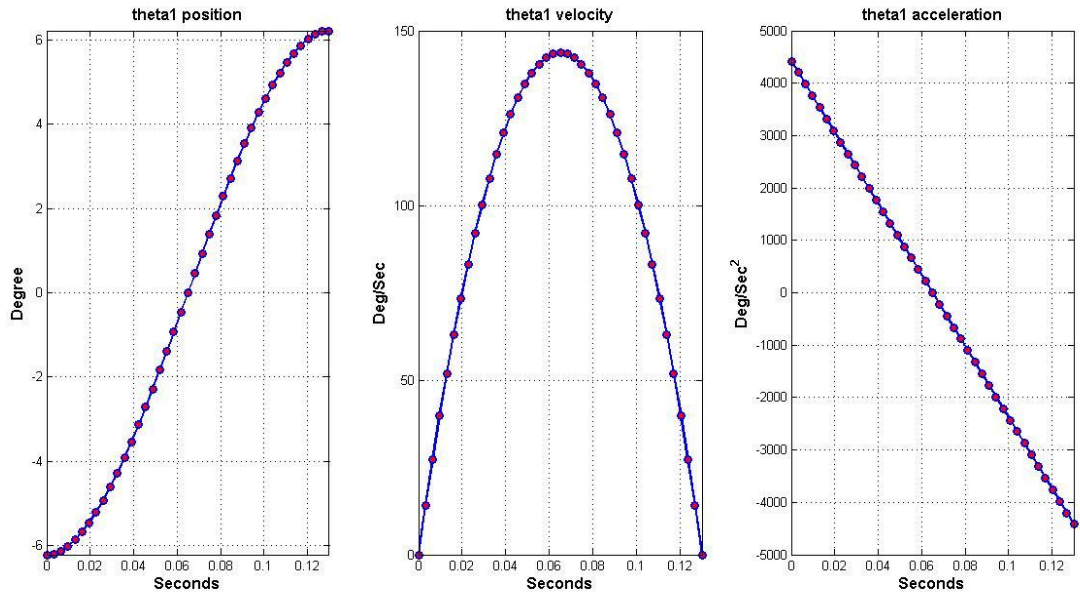

Fig.10 Coxa angle trajectory
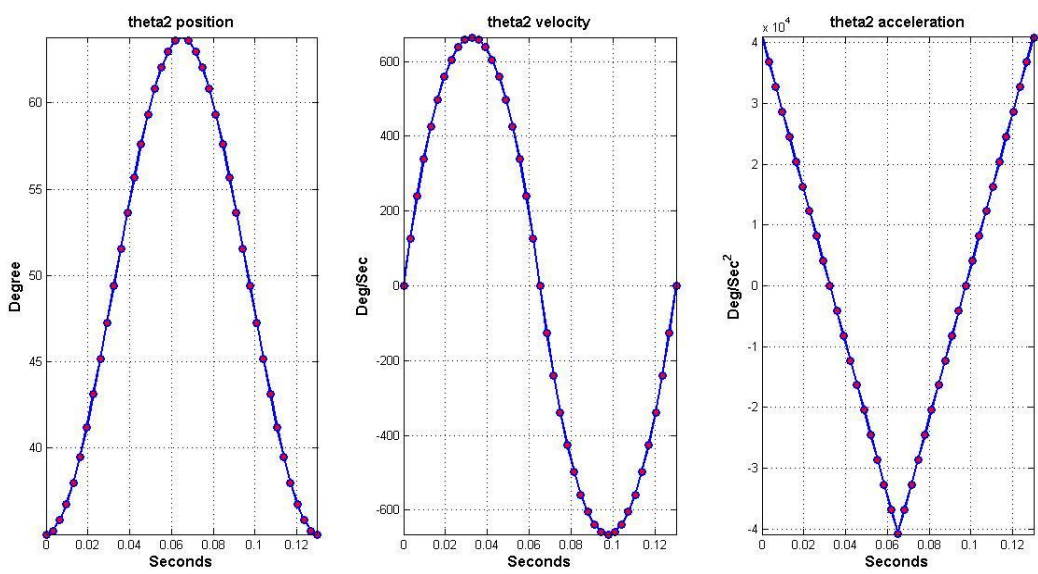

Fig. 11 Femur angle trajectory
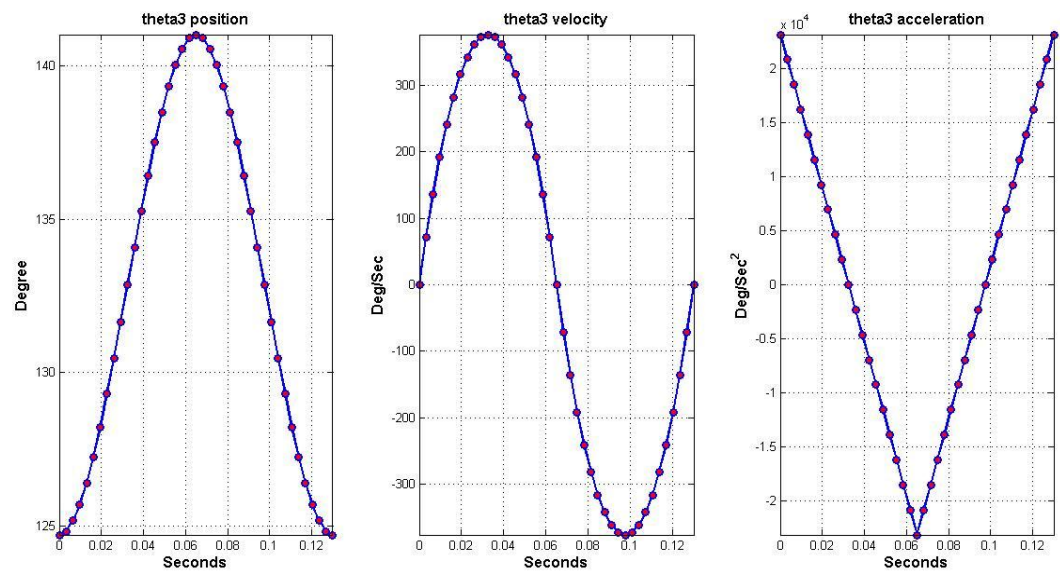

Fig. 12 Tibia angle trajectory

Disadvantage of cubic paths is the acceleration jump at boundaries that introduces infinite jerks so we will use another method. 


\section{Quantic polynomial}

For the process of trajectory planning the quantic polynomials are chosen in the form

$$
\begin{aligned}
& \theta(t)=a_{0}+a_{1} t+a_{2} t^{2}+a_{3} t^{3}+a_{4} t^{4}+a_{5} t^{5} \\
& \theta \cdot(t)=a_{1}+2 \times a_{2} t+3 \times a_{3} t^{2}+4 \times a_{4} t^{3}+5 \times a_{5} t^{4} \\
& \theta \cdot(t)=2 \times a_{2}+6 \times a_{3} t+12 \times a_{4} t^{2}+20 \times a_{5} t^{3}
\end{aligned}
$$

Here we have rest-to-rest path with no acceleration at the rest positions with the following conditions:

$$
\begin{aligned}
& \theta(0)=\theta_{0}, \theta \cdot(0)=0, \theta \cdot(0)=0 \\
& \theta\left(t_{f}\right)=\theta_{f}, \theta \cdot\left(t_{f}\right)=0, \theta \cdot\left(t_{f}\right)=0
\end{aligned}
$$
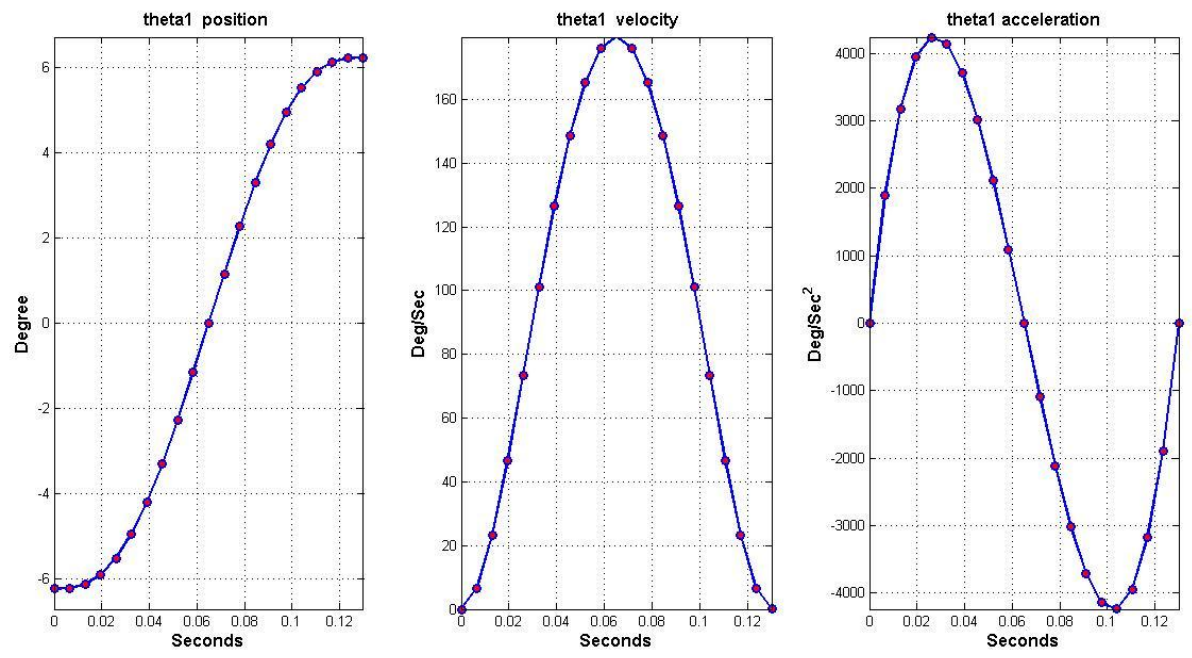

Fig. 13 Coxa angle trajectory
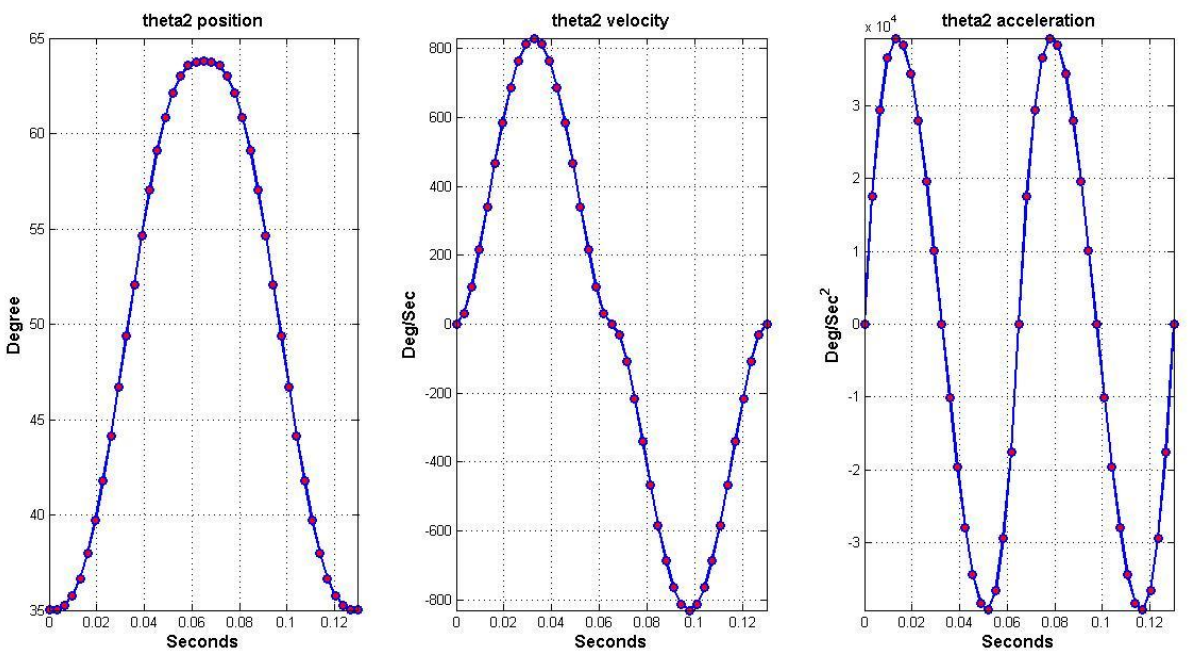

Fig. 14 Femur angle trajectory 

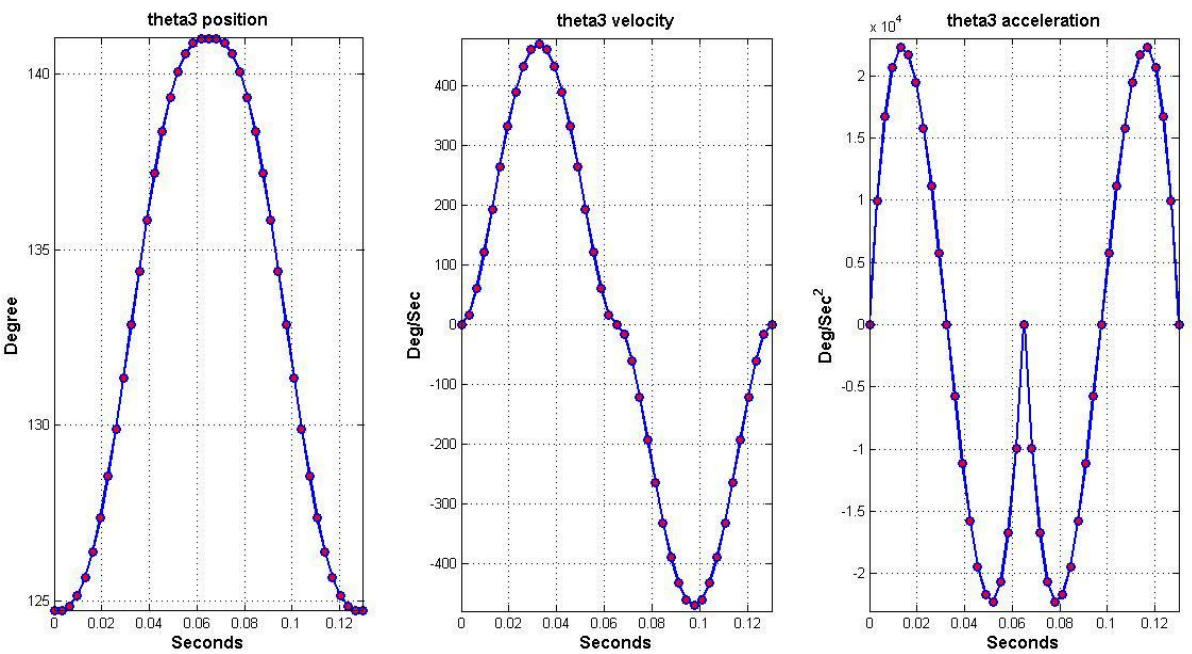

Fig. 15 Tibia angle trajectory

The motion is forced to have zero acceleration and zero jerk at start and stop. Hence, it shows the smoothest start and stop.

\section{Support Phase}

During support phase the robot stands on the swing legs and move the body in the direction of motion(forward, backward, sideward or rotational) when the body move it is only changes the values of $\theta_{1}$ for all the legs and the values of $\theta_{2}$ and $\theta_{3}$ remains constant. Here we will use a quantic polynomial with rest to rest conditions at the start and end of the path as seen in Fig. 16.
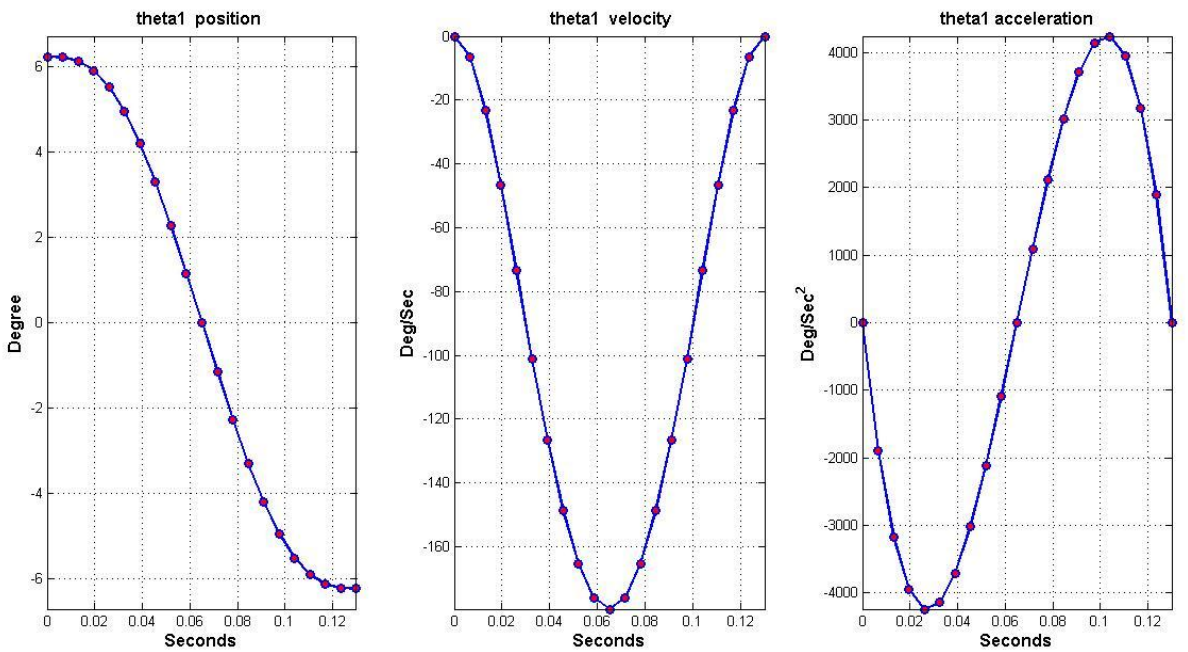

Fig. 16 Body motion during support phase 


\section{Validation of Results of Leg Trajectory}

To validate our calculation we compare the results with the results in [6], Table 3 contains the values of joint angle, and Fig.17 illustrates simulation results and the results of quantic polynomial are shown in Fig. 18.

Table 3 Initial and final values

of joint angles

\begin{tabular}{|c|c|c|c|}
\hline Point/ & $\theta_{1}$ & $\theta_{2}$ & $\theta_{3}$ \\
\hline start point $\theta_{0}$ & 345 & 310 & 4 \\
\hline via point $\theta_{\mathrm{v}}$ & 355 & 330 & 34 \\
\hline end point $\theta_{\mathrm{g}}$ & 365 & 310 & 4 \\
\hline
\end{tabular}
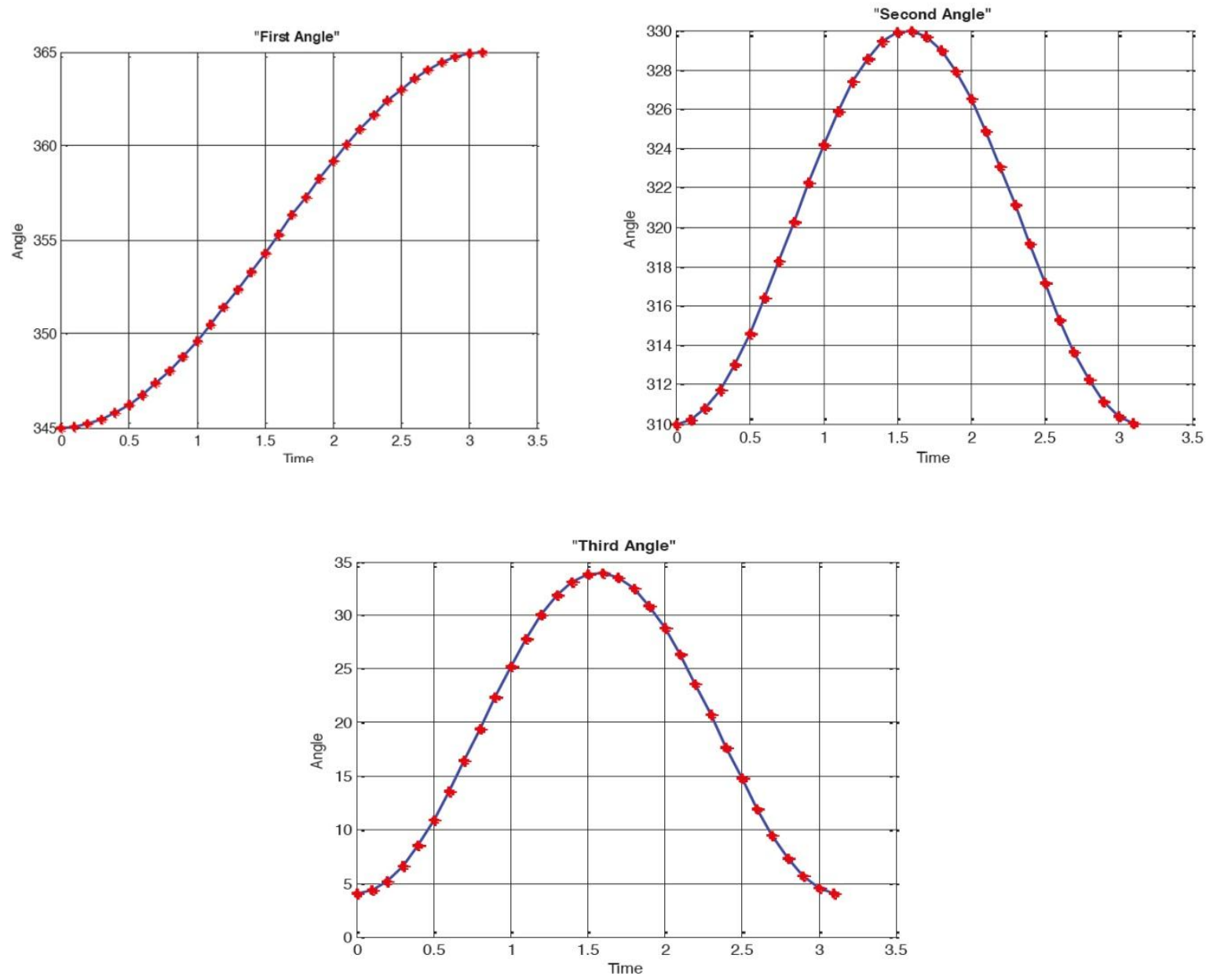

Fig. 17 Simulation results[6] 

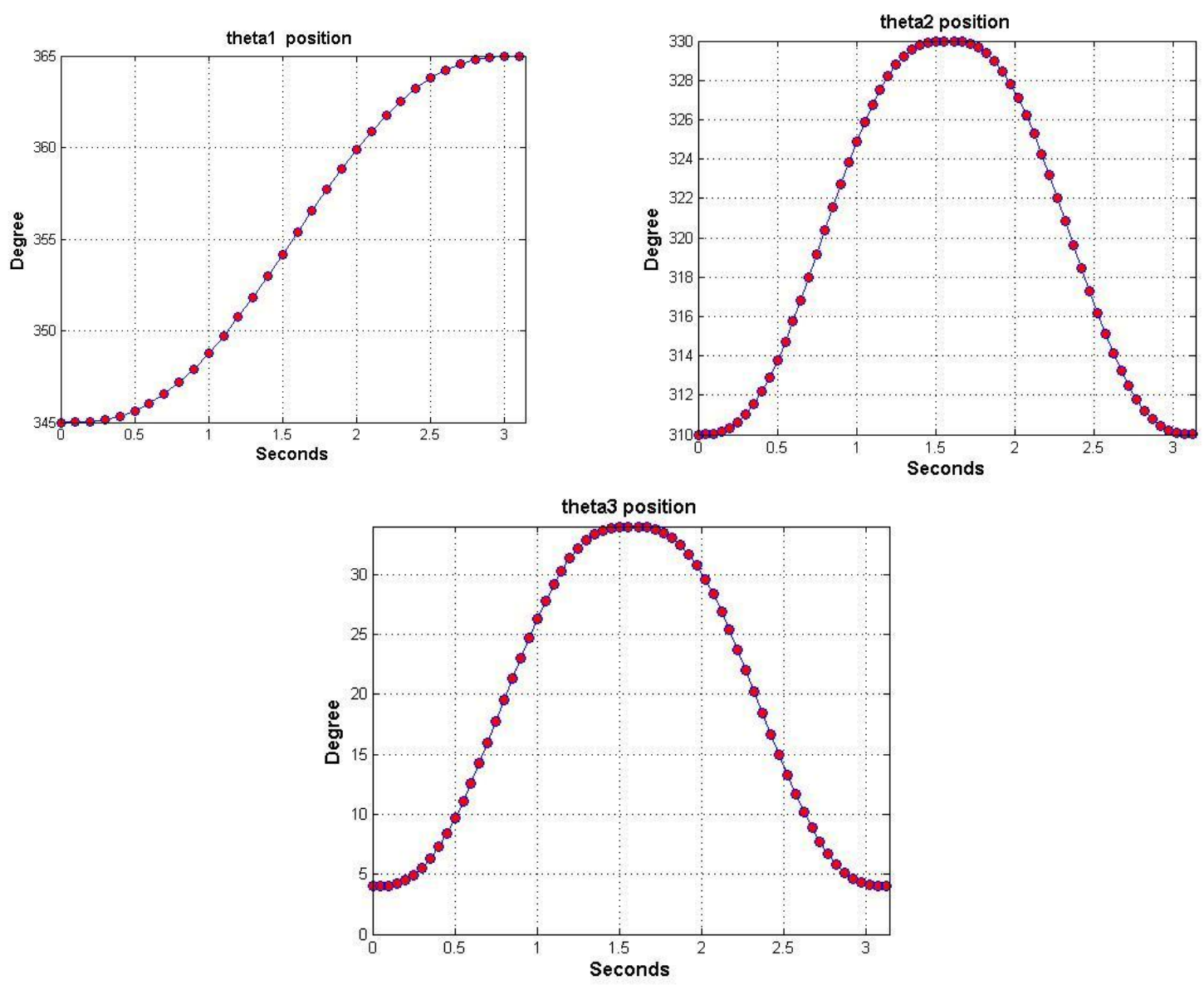

Fig. 18 current model results

\section{Conclusions}

The proposed kinematic model shows ability of modeling phantom AX12 hexapod robot since output control commands are continuous and differentiable functions. Given the position of leg tip, all joint variables are then calculated graphically using inverse kinematics and the results are visualized for the sake of validation. Cubic polynomial trajectory gives zero initial and finial velocities of leg tip at the desired points determined by forward kinematic analysis. Quantic polynomials prove its ability to perform a smooth trajectory with both zero velocities and acceleration at initial and final leg configurations. Leg trajectory model results are then compared to come previously publish research and gives even smoother motion in case of quantic functions.

\section{References}

[1] E. Burkus and P. Odry, "Autonomous Hexapod Walker Robot," in Intelligent Systems and Informatics, 2007. SISY 2007. 5th International Symposium on, 2007, pp. 103-106.

[2] M. J. C. Ronde, "Simulating the "first steps" of a walking hexapod robot," Master's thesis Master's thesis, Department Mechanical Engineering Control Systems Technology Group, Technische Universiteit Eindhoven, Eindhoven, January, 2011.

[3] X. Ding, Z. Wang, A. Rovetta, and J. Zhu, "Locomotion analysis of hexapod robot," Climbing and Walking Robots, pp. 291-310, 2010.

[4] J. J. Craig, Introduction to Robotics, 2005.

[5] R. P. Mads Jensen, Jeppe Juel Petersen,Casper Lyngesen Mogensen,Henrik Dalsager Christensen, "Modelling and Control of a Hexapod Robot," Project2nd of June, 2009. 
[6] M. García-López, E. Gorrostieta-Hurtado, E. Vargas-Soto, J. Ramos-Arreguín, A. Sotomayor-Olmedo, and J. Morales, "Kinematic analysis for trajectory generation in one leg of a hexapod robot," Procedia Technology, vol. 3, pp. 342-350, 2012. 